\title{
Correlation of BCRP Expression to NPM1 Mutations in Acute Myeloid Leukemia
}

\author{
Tayebeh SOHRABI ${ }^{1}$, Hossein AYATOLLAHI ${ }^{2}$, Mohsen ROKNI ${ }^{3}$, Samaneh HOUSHMAND ${ }^{1}$, \\ Zahra ZAFARI ${ }^{1}$, Mohammad H. SADEGHIAN ${ }^{2}$, Mohammad R. KERAMATI ${ }^{2}$
}

\footnotetext{
${ }^{1}$ Mashhad University of Medical Sciences, Faculty of Medicine, Department of Hematology and Blood Bank, Mashhad

${ }^{2}$ Mashhad University of Medical Sciences, Cancer Molecular Pathology Research Center, Mashhad

${ }^{3}$ Tehran University of Medical Sciences, School of Public Health, Department of Immunology, Tehran, IRAN
}

\begin{abstract}
Nucleophosmin1 (NPM1) mutation is a new prognostic factor for acute myeloid leukemia. Significant complete remission after chemotherapy has been observed in patients with this mutation compared to non-mutated patients. Overexpression of breast cancer resistance protein (BCRP) is directly associated with multiple drug resistance in leukemia. The aim of this study was to identify the effect of normal and mutated NPM1 on the expression of BCRP in AML patients. This cross-sectional study was conducted on 80 AML patients and 20 healthy individuals as the control group between 2012 and 2017. NPM1 mutations were observed with the PCR technique and the BCRP expression was measured by real-time PCR. Screening of the 80 AML patients revealed NPM1 mutation in $9(11.2 \%)$ of them. The average expression of BCRP decreased significantly in the AML patient group in com-parison with the control group $(p<0.001)$. No statistically significant difference was observed between NPM1 mutation and BCRP expression in both groups with and without NPM1 mutations $(p=0.394)$. Unlike other studies, no case of NPM1 mutation was reported in the M3 subtype. However, in this study, this mutation was observed in the M3 subtype, and should be investigated for genetic and racial factors' implication. There was no correlation between NPM1 mutation and BCRP expression. There are independently prognostic and treatment management factors in AML patients, which should be examined and considered in order to determine the treatment process and evaluation of drug resistance.
\end{abstract}

Keywords: Acute myeloid leukemia, NPM1, BCRP, Real-Time PCR

\section{INTRODUCTION}

Acute myeloid leukemia (AML) is a malignant and neoplastic disease of hematopoietic tissue induced by autonomous proliferation and defective differentiation of hematopoietic precursor cells. It is marked by the accumulation of immature myeloid cells in the bone marrow. ${ }^{1}$ There are numerous genetic alterations that are associated with the onset of leukemia such as point mutations, genes rearrangement and chromosomal translocations. ${ }^{2}$ On the basis of age, morphological and cytogenetic abnormalities, there are differences in prognosis of AML patients after chemotherapy. Gene mutation in nucleophosmin 1 (NPM1) is a new agent that is imperative in the treatment and prognosis of AML patients. ${ }^{3}$ Patients with the NPM1 mutation have a favorable prognosis and significant complete remission (CR) after chemotherapy compared with non-mutated patients. However, the molecular basis of these observations remains unclear presently. ${ }^{4,5}$ NPM1 gene is located on chromosome $5 q 35$ and contains 12 exons. 
Its main product is a 294 amino acid phosphoprotein that belongs to the nuclear chaperone family, and is mostly concentrated in the nucleolus and constantly moving between the nucleus and cytoplasm. It can be noted that the most significant functions of the NPM1 are displacement of ribosomal components into the cytoplasm, control of centrosome duplication and RNA binding. ${ }^{1,6}$ Approximately 50 molecular variants of NPM1 mutations have been identified so far; most of these mutations usually occur at the C-terminal of NPM1 exon-12. The arrangement of the NPM1 mutations at the C-terminal of NPM1 exon-12 leads to ectopic accumulation of mutant NPM1 in the cytoplasm of leukemic cells (NPMc+). ${ }^{7}$ Breast cancer resistance protein (BCRP) is a member of the $\mathrm{ABC}$ superfamily and has been identified as a prognostic factor. This protein is a membrane transporter that removes a wide range of chemotherapy drugs from leukemic cells and induces the inactivation of chemotherapy. The overexpression of BCRP is directly associated with multiple drug resistance (MDR), high rate of relapse and poor prognosis in AML patients; and it can be a therapeutic target for decreasing drug resistance to chemotherapy in AML patients. ${ }^{89}$. BCRP expression is activated by NF- $\kappa \mathrm{B}$ through direct DNA binding and down regulation following decrease in NF- $\kappa \mathrm{B}$ activity. This mechanism may enhance sensitivity to some chemotherapy drugs. ${ }^{10}$ However, there is little available knowledge about the possible mechanisms affecting the expression of BCRP in AML patients. ${ }^{8}$ Additionally, mutated NPM1 also binds to NF- $\mathrm{KB}$ and its retention in the cytoplasm leads to inactivation and decreased levels of NF- $\kappa B$ in

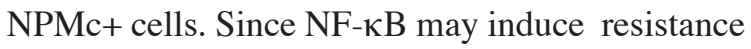
to chemotherapy in AML patients, this mechanism may cause a high sensitivity of AML-NPMc+ patients to chemotherapy. ${ }^{4,11}$ The aim of this study was to identify the frequency of NPM1 mutations and the effect of normal and mutant NPM1 gene on the expression of BCRP in AML patients.

\section{PATIENTS AND METHODS}

\section{Patients and data collection}

This cross-sectional study was conducted in the Cancer Molecular Pathology Research Center between March 2012 and March 2017 and the Local
Ethics Committee approved the research protocol. A total of 80 patients who were diagnosed with AML, and 20 healthy individuals that had no neoplastic disorders in their history or peripheral blood and bone marrow samples were included in this study. Diagnosis of AML was based on standard morphological and immunophenotypical criteria as specified in the World Health Organization (WHO) classification. AML patients who were undergoing chemotherapy or had recur $\neg$ rent leukemia, uncertain diagnosis and inappropriate samples were excluded from the study.

\section{Detection of NPM1 Mutations Using PCR}

Total RNA was extracted from mononuclear cells of the peripheral blood and bone marrow samples using the TriPure Isolation Reagent Kit (Roche Diagnostic, Germany) and cDNA was synthesized employing the RevertAidTM cDNA Synthesis Kit (Fermentas, Germany). Afterwards, NPM1mutations were detected employing the polymerase chain reaction (PCR) method proposed by Oppliger Leibundgut et al. ${ }^{12}$, (10x PCR buffer II, $\mathrm{MgCl} 2$, dNTPs, Taq DNA polymerase (CinnaGen, Iran), cDNA (1:50), Thermal Cycler Applied Biosystems). Reaction conditions consisted of an initial denaturation step at $94^{\circ} \mathrm{C}$ for $10 \mathrm{~s}$ followed by 35 cycles at $94^{\circ} \mathrm{C}$ for $0 \mathrm{~s}, 60^{\circ} \mathrm{C}$ for $5 \mathrm{~s}$, and $72^{\circ} \mathrm{C}$ for $20 \mathrm{~s}$ and a final elongation step at $72^{\circ} \mathrm{C}$ for 30 s. Amplified products were electrophoresed in $8 \%$ polyacrylamide gel and visualized under UV light after staining with ethidiumbromide. If a double band is seen in the range of $446 \mathrm{bp}$, it indicates a heterozygote mutation and a single band represents the wild type sequence (Figure 1).

\section{Real-Time PCR and BCRP Gene Expression}

After extraction of total RNA, synthesis of cDNA and PCR of the GAPDH (glyceraldehyde 3-phosphate dehydrogenase) gene for these cDNAs, there was an assurance that the experimental process had been done ideally. Thereafter, the real-time qPCR technique was carried out to evaluate the BRCP mRNA expression using SYBR Premix Ex TaqTM II kit (Takara, Japan) on an ABI thermocycler (One Step, USA). Parameters of the real-time qPCR were established according to the protocol of the kit employed (Takara, Japan). Primers utilized are 


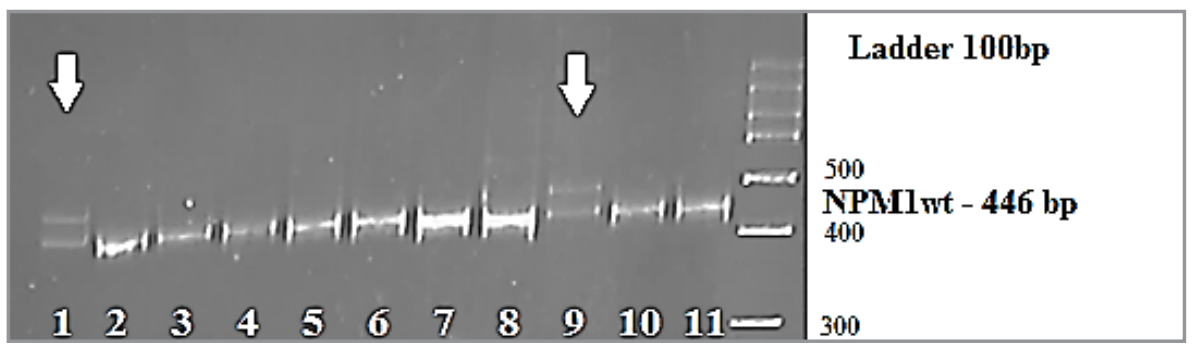

Figure 1. Electrophoresis results in a number of AML patients. Patient specimens No.1 and 9 are positive for NPM1 mutation (double-band).

shown in Table 1. In order to match the concentration of the cDNAs produced, dilution of cDNA with distilled water without DNase was carried out. The GAPDH gene was employed as the reference gene (a housekeeping gene) for standardization of the mRNA level of BCRP. The result of the BCRP mRNA expression was analyzed by the $2^{-\triangle \Delta C T}$ method. Prior to analysis, the efficiency of both GADPH and BCRP was determined employing dilution series. The specificity of the real-time qPCR products was confirmed by the melt curve analysis.

\section{Statistical Analysis}

Data analysis was performed using SPSS software, version 11.5. Descriptive and compara $\neg$ tive analyses of parameters were initially performed. The non-parametric Mann-Whitney test was used to compare the mean of gene expression between the positive and negative mutation patient groups and the $\chi 2$ or Fisher exact (whenever an expected value was $<5$ ) test for categorical data was employed to compare demographic, laboratory and molecular parameters between the patient groups due to the non-normal distribution of mutations and expression values. A p-value $\leq 0.05$ was considered to be statistically significant.

\section{RESULTS}

After exclusion of the nine samples, 80 patients diagnosed with AML and 20 healthy individuals as the control group were analyzed. In the patient group, $44(55 \%)$ were male and $36(45 \%)$ were female, while in the control group, $10(50 \%)$ were male and $10(50 \%)$ were female. There was no significant difference in the number of sexes in the two groups $(\mathrm{p}=0.688)$. The mean age $( \pm \mathrm{SD})$ was $29 \pm$ 18.7 and $29 \pm 18.5$ years and the age range was 1 month - 79 years and 1- 77 years in the patient and control groups, respectively. The mean age difference between the two groups was not significant $(\mathrm{p}=0.954)$. Twenty-two $(27.5 \%)$ patients were children under 15 years, while sixteen $(72.7 \%)$ of them were male $(\mathrm{p}=0.05)$. Mean of White Blood Cell counts, Platelet count and Hemoglobin concentration were significantly different in the AML patients and normal control $(\mathrm{p}<0.001)$. Among the AML patients, the most common subtypes (according to the FAB classification) were M2 and M3 with a frequency of $31.2 \%$ and $30 \%$, respectively. Forty-nine $(61.2 \%)$ patients had no chromosomal abnormalities (NK AML) and the highest cytogenetic abnormality was observed in $\mathrm{t}(15 ; 17)$ (PMLRARA) with a frequency of $27.5 \%$. Screening of the 80 AML patients showed NPM1 mutations in

\begin{tabular}{|lll|}
\hline \multicolumn{2}{|l|}{ Table 1. Primer sequence used for target genes } \\
\hline Genes & Sequences \\
\hline NPM1 & F & 5'-GCGCCAGTGAAGAAATCTATAC-3' \\
& R & 5'-GGACAACATTATCAAACACGG-3' \\
BCRP & F & 5'-CGTGGCCTTGGCTTGTATGATTGTA-3' \\
& R & 5'-GGCAAGGGAACAGAAACAACAAAA-3' \\
GAPDH & F & 5'-GCCCCAGCAAGAGCACAAGAGGAAGA-3' \\
& R & 5'-CATGGCAACTGTGAGGAGGGAGAT-3' \\
\hline
\end{tabular}


International Jounal of Hematology and Oncology

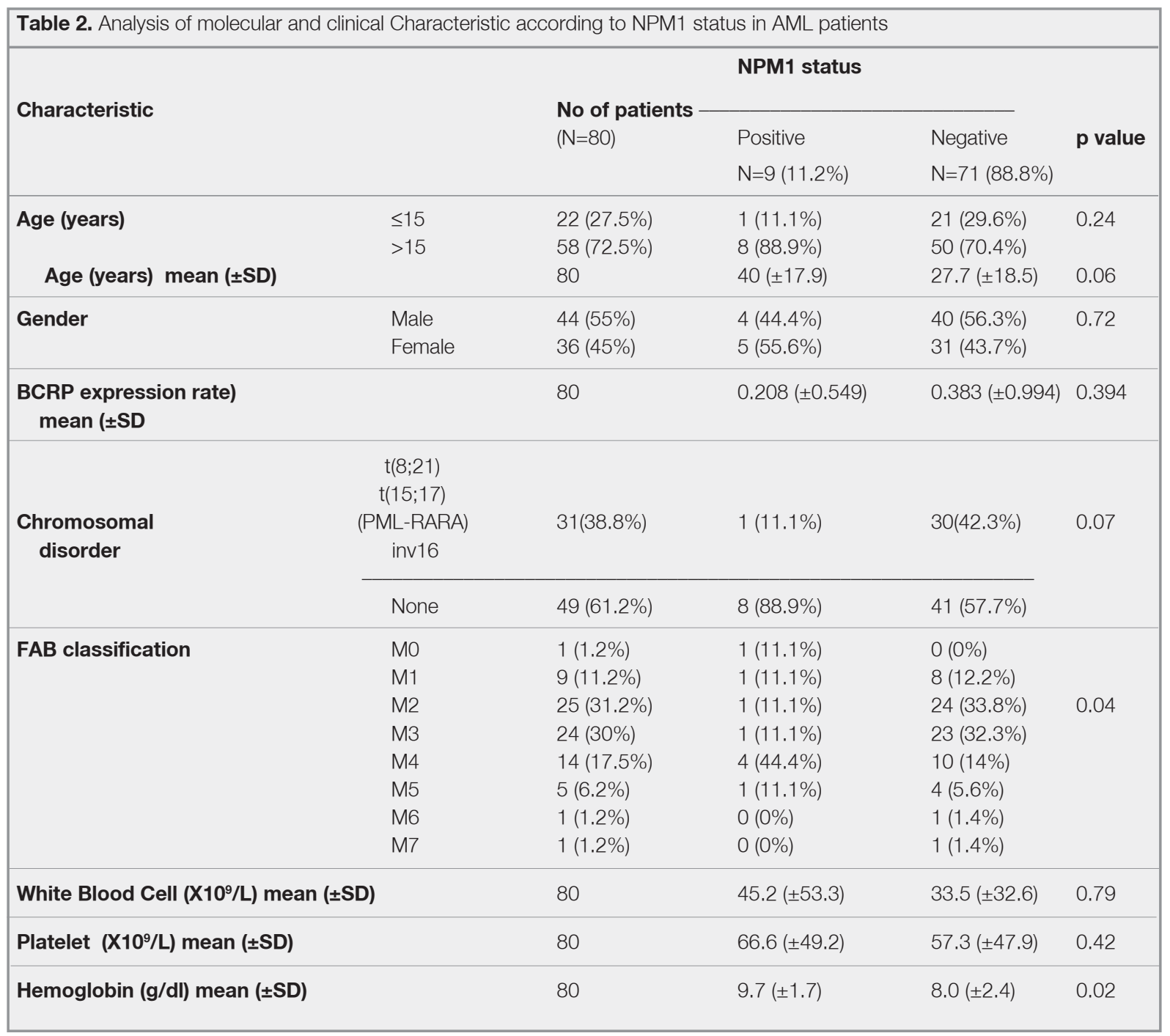

$9(11.2 \%)$ of them (Table 2). NPM1 mutation was observed in 8 of 49 (16.3\%) NK AML patients. There was no statistically significant difference in the average age of patients with and without NPM1 mutations $(40 \pm 17.9$ vs. $27 \pm 18.5$ years, respectively; $\mathrm{p}=0.06)$. However, the results obtained were close to the statistical significance level such that the mean age was higher in patients with NPM1 mutations. The frequency of NPM1 mutations did not show any significant difference between NPM1 mutation and gender $(\mathrm{p}=0.724)$. In this study, the highest frequency of NPM1 mutations was observed in the M4 subtype (44.4\%) compared to other subtypes (M0, M1, M2, M3, M5 $=11.1 \%$; $\mathrm{p}=0.04)$. There was no NPM1 mutation in the M6 and M7 subtypes. The mean of WBC, Plt and $\mathrm{Hb}$ were assessed in both patients with and without
NPM1 mutation. There was no significant difference between the two groups in WBC and Plt levels; however, patients with NPM1 mutations had a significantly higher mean value of $\mathrm{Hb}$ compared to patients without NPM1 mutation $(9.7 \mathrm{~g} / \mathrm{dLvs} .8 .0 \mathrm{~g} /$ $\mathrm{dL} ; \mathrm{p}=0.02$ ) (Figure 2).

The average expression level of BCRP in the patient group when compared with the control group was $0.363 \pm 0.954$ (about 3-fold down-regulation), which indicates a significant reduction in the expression level of BCRP $(\mathrm{p}<0.001)$ (Table 3$)$. Among these, 12 (15\%) of 80 AML patients had upregulation of BCRP. Major cases of reduction in BCRP expression (84.8\%) were more prevalent in patients with WBC count less than $50 \times 10^{9} / \mathrm{L}$ compared to patients with WBC count more than $50 \times 10^{9} / \mathrm{L}(\mathrm{p}=0.03)$. There was no difference 


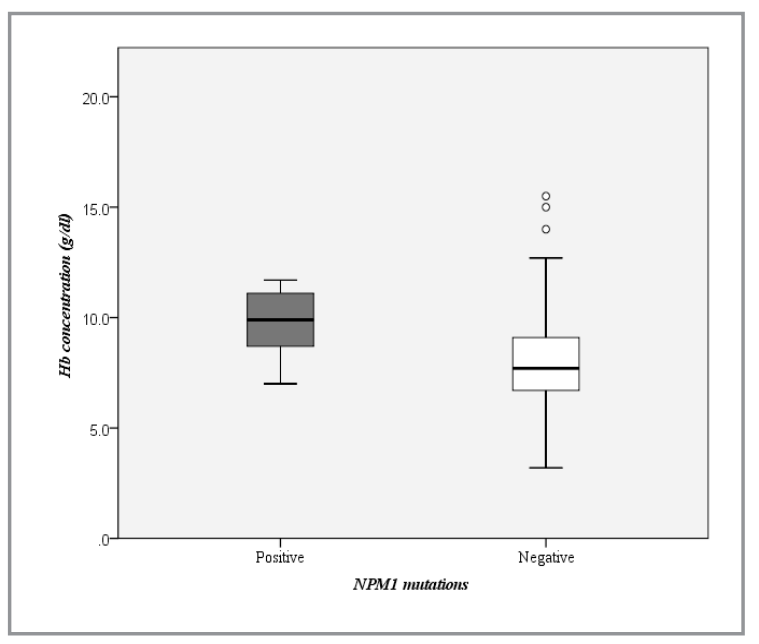

Figure 2. Box plot of mean $\mathrm{Hb}$ concentration in $\mathrm{AML}$ patients with and without NPM1 mutations $(9.7 \mathrm{~g} / \mathrm{dL}$ vs. $8.0 \mathrm{~g} / \mathrm{dL} ; \mathrm{p}=$ 0.02).

in BCRP expression between age, gender, FAB classification and chromosomal disorder. No statistically significant difference was observed between NPM1 mutation and the average expression level of BCRP in the two groups with and without NPM1 mutations $(0.208 \pm 0.549$ vs. $0.383 \pm 0.994$, respectively; $\mathrm{p}=0.394)$.

\section{DISCUSSION}

NPM1 mutations are imperative in the treatment and prognosis of acute myeloid leukemia in patients with the disease, and BCRP can be a therapeutic target for reducing drug resistance to chemotherapy in AML. ${ }^{3,89}$ In this study, the relationship between NPM1 mutation and BCRP expression levels was examined in order to comprehend the underlying cause of the high sensitivity of patients with NPM1 mutation to chemotherapy, and assess the adverse prognosis in patients without NPM1 mutation. NPM1 mutations has an incidence rate of $25-35 \%$ in one-third of adults with AML. ${ }^{13}$ Sundry frequencies of NPM1 mutations have been reported in different populations. In this study, the prevalence of NPM1 mutations was $11.2 \%$ in 80 AML patients. The prevalence of NPM1 mutations in India (19.5\% of 200 patients), Taiwan (19.1\% of 173 patients), Germany (27.5\% of 1485 patients) and Italy (35.2\% of 591 patients) have been reported. ${ }^{5,14-16}$ The frequency of mutation in this study was relatively low compared to other studies conducted elsewhere in the world due to the differences in sample size, age of patients and the low prevalence of NPM1 mutations in children, which were efficacious in the study results. Several studies such as Verhaak ${ }^{17}$, Falini ${ }^{14}$, Chauhan ${ }^{18}$ and Chou $^{15}$ have shown that the frequency of NPM1 mutations increased in older adults. High incidences of NPM1 mutations (22 to $38 \%$ ) have been reported in patients over 40 years and rarely observed in patients less than 40 years; and its frequency have been reported to be low in children. In the present study, patients with NPM1 mutations had a higher average age than non-mutated patients; this finding was similar to the results of other studies. Most of the patients $(69.3 \%)$ were under the age of 40 years; this was targeted at reducing the chance of finding NPM1 mutations. As expected, the frequency of NPM1 mutations in adults (13.8\%) was higher than children $(4.5 \%)$. Since $27.5 \%$ of the patients were children, the lower frequency of NPM1 mutation observed in children was one of the other reasons that decreased the possibility of finding NPM1 mutation in the study. The major NPM1 mutations (88.9\%) were observed in patients with normal karyotype and no genetic disorders, which indicate the leukemogenesis of this mutation in AML patients with normal karyotype. ${ }^{19}$ In the study of Zaker et al. ${ }^{20}$, the NPM1 mutation was observed to

\begin{tabular}{|llll|}
\hline \multicolumn{2}{|l|}{ Table 3. Laboratory characteristics of AML patients compared to control group } \\
\hline Characteristic & $\begin{array}{l}\text { Patient group } \\
\text { mean }( \pm \text { SD) }\end{array}$ & $\begin{array}{l}\text { Control group } \\
\text { mean }( \pm \text { SD) }\end{array}$ & p-value \\
\hline BCRP FOLD change & $0.363( \pm 0.954)$ & $1.0( \pm 0.0)$ & $<0.001$ \\
White Blood Cell (X10\%/L) & $34.8( \pm 35.3)$ & $7( \pm 1.3)$ & $<0.001$ \\
Hemoglobin (g/dl) & $8.2( \pm 2.3)$ & $13.6( \pm 1.7)$ & $<0.001$ \\
Platelet $\left(X 10^{\prime} / \mathrm{L}\right)$ & $58.3( \pm 47.9)$ & $256( \pm 61)$ & $<0.001$ \\
\hline
\end{tabular}


be more prevalent in the M4 subtype and no mutation was observed in the M1 and M7 subtypes. Falini et al. ${ }^{14}$, reported the highest frequency in the M5 subtype and this mutation was not observed in M3 and M7 subtypes. In other studies, this mutation was observed mainly in M5 and M2 patients and no cases of NPM1 mutation was detected in the M3 subtype. ${ }^{2,5}$ In the present study, the highest frequency of NPM1 mutations in the monocytic subtypes (M4 and M5) was in consonance with the results obtained from other studies. Unlike other studies, no case of NPM1 mutation was reported in the M3 subtype. In this study, this mutation was observed in the M3 subtype; this should be investigated for possible genetic and racial factors' implication. ${ }^{2,5,14}$ AML patients in this study showed a $15 \%$ upregulation in BCRP expression, which is almost lower than the results obtained in other studies. Ross et al. ${ }^{21}$, Abbott et al. ${ }^{22}$, and Sargent ${ }^{23}$ reported 33\%, $7 \%$ and $27 \%$ increase in BCRP expression, respectively. Decreased BCRP expression has many potential causes. For example, in AML samples, in addition to leukemic blast, there are also normal hematopoietic cells (erythroid progenitors, NK cells, primary stem cells) that reduce the expression of BCRP due to their percentage. ${ }^{24}$ On the other hand, it has been observed in studies that BCRP proteins are naturally expressed in a subset of hematopoietic stem cells (SP) with immature phenotypes. The percentage of these cells varies in the bone marrow of AML patients. These cells (SP) are leukemic and non-leukemic in the bone marrow of patients, and the rate of BCRP expression in leukemic cases is more than non-leukemic. The reduction in BCRP expression may be due to the different percentage of these cells in the sample of AML patients. ${ }^{22,25,26}$

Other studies conducted to investigate the association of this mutation with BCRP expression are limited, and only one study by Palaiologou et al., was performed on 38 AML patients (15 of them NPM1 positive and 23 of the patients were without this mutation). ${ }^{27}$ The results of this study indicated a significantly higher BCRP expression in NPM1 negative patients $(p<0.0001)$. However, in the present study, there was no significant correlation between NPM1 mutation and BCRP expression levels $(\mathrm{p}=0.394)$. This could be adduced to the low number of mutations found and the sample size used for the study in comparison with our studies. In conclusion, there was no correlation between NPM1 mutation and BCRP expression. There are independently prognostic and treatment management factors in AML patients, which should be examined and considered in order to determine the treatment process, evaluation of drug resistance and MRD.

\section{Acknowledgement:}

We would like to thank the management and staff of Cancer Molecular Pathology Research Center of Ghaem Hospital for their cooperation. There was no conflict of interest in this study.

\section{REFERENCES}

1. Ghosh K, Swaminathan S, Madkaikar M, et al. FLT3 and NPM1 mutations in a cohort of AML patients and detection of a novel mutation in tyrosine kinase domain of FLT3 gene from Western India. Ann Hematol 91: 1703-1712, 2012.

2. Suzuki T, Kiyoi H, Ozeki K, et al. Clinical characteristics and prognostic implications of NPM1 mutations in acute myeloid leukemia. Blood 106: 2854, 2005.

3. Sanaat Z, Shams K, Nejati B, et al. NPM1 and FLT3-(ITD) Gene mutations and laboratory findings in patients with acute myeloid leukemia in Northwest of Iran. J Gorgan Univ Med Sci 16: 80-86, 2014.

4. Cilloni D, Messa F, Rosso V, et al. Increase sensitivity to chemotherapeutical agents and cytoplasmatic interaction between NPM leukemic mutant and NF-kappaB in AML carrying NPM1 mutations. Leukemia 22: 1234-1240, 2008.

5. Thiede C, Koch S, Creutzig E, et al. Prevalence and prognostic impact of NPM1 mutations in 1485 adult patients with acute myeloid leukemia (AML). Blood 107: 4011-20, 2006.

6. Kazem AH, Mikhael IL, Ghanem AM. Cytoplasmic nucleophosmin (cNPM) in acute myeloid leukaemia: Relation to disease characteristics. AJM 47: 225-235, 2011.

7. Falini B, Gionfriddo I, Cecchetti F, et al. Acute myeloid leukemia with mutated nucleophosmin (NPM1): any hope for a targeted therapy? Blood Rev 25: 247-254, 2011.

8. Nasilowska-Adamska B, Solarska I, Paluszewska M, et al. FLT3-ITD and MLL-PTD influence the expression of MDR-1, MRP-1, and BCRP mRNA but not LRP mRNA assessed with $\mathrm{RQ}-\mathrm{PCR}$ method in adult acute myeloid leukemia. Ann $\mathrm{He}$ matol 93: 577-593, 2014.

9. Entezar-e-Ghaem M, Rahgozar S, Moafi A, et al. Evaluation of mRNA expression profile of ABCG2/BCRP in childhood acute lymphoblastic leukemia. Shahid Sadoughi Univ Med Sci (JSSU) 21: 575-586, 2013. 
10. Wang $X$, Wu X, Wang C, et al. Transcriptional suppression of breast cancer resistance protein (BCRP) by wild-type p53 through the NF-kappaB pathway in MCF-7 cells. FEBS Lett 584: 3392-3397, 2010.

11. Falini B, Bolli N, Liso A, et al. Altered nucleophosmin transport in acute myeloid leukaemia with mutated NPM1: molecular basis and clinical implications. Leukemia 23: 1731-1743, 2009.

12. Oppliger LE, Porret NA, Bienz MM, et al. Rapid and highly specific screening for NPM1 mutations in acute myeloid leukemia. Anna Hematol 92: 173-177, 2013.

13. Falini B. Acute Myeloid Leukemia with Mutated Nucleophosmin (NPM1): Molecular, Pathological, and Clinical Features. In: Nagarajan L, editor. Acute Myelogenous Leukemia: Springer, 2010: 149-168.

14. Falini B, Mecucci C, Tiacci E, et al. Cytoplasmic nucleophosmin in acute myelogenous leukemia with a normal karyotype. N Eng J Med 352: 254-266, 2005.

15. Chou W-C, Tang J-L, Lin L-I, et al. Nucleophosmin mutations in de novo acute myeloid leukemia: the age-dependent incidences and the stability during disease evolution. Cancer Res 66: 3310-3316, 2006

16. Ahmad F, Mandava S, Das BR. Mutations of NPM1 gene in de novo acute myeloid leukaemia: determination of incidence, distribution pattern and identification of two novel mutations in Indian population. Hematol Oncol 27: 90-97, 2009.

17. Verhaak RG, Goudswaard CS, van Putten W, et al. Mutations in nucleophosmin (NPM1) in acute myeloid leukemia (AML): association with other gene abnormalities and previously established gene expression signatures and their favorable prognostic significance. Blood 106: 3747-3754, 2005.

18. Chauhan PS, Ihsan R, Singh L, et al. Mutation of NPM1 and FLT3 genes in acute myeloid leukemia and their association with clinical and immunophenotypic features. Dis Markers 35: 581-588, 2013.

19. Grisendi S, Mecucci C, Falini B, Pandolfi PP. Nucleophosmin and cancer. Nat Rev Cancer 6: 493-505, 2006.

20. Pazhakh V, Zaker F, Alimoghaddam K, Atashrazm F. Detection of nucleophosmin and FMS-like tyrosine kinase-3 gene mutations in acute myeloid leukemia. Ann Saudi Med 31: 4550, 2011.

21. Ross DD, Karp JE, Chen TT, Doyle LA. Expression of breast cancer resistance protein in blast cells from patients with acute leukemia. Blood 96: 365-368, 2000.

22. Abbott BL, Colapietro A-M, Barnes $\mathrm{Y}$, et al. Low levels of ABCG2 expression in adult AML blast samples. Blood 100: 4594-601, 2002.

23. Sargent JM, Williamson CJ, Maliepaard M, et al. Breast cancer resistance protein expression and resistance to daunorubicin in blast cells from patients with acute myeloid leukaemia. Br J Haematol 115: 257-262, 2001.
24. Scharenberg CW, Harkey MA, Torok-Storb B. The ABCG2 transporter is an efficient Hoechst 33342 efflux pump and is preferentially expressed by immature human hematopoietic progenitors. Blood 99: 507-512, 2002.

25. Feuring-Buske M, Hogge DE. Hoechst 33342 efflux identifies a subpopulation of cytogenetically normal CD34+ CD38progenitor cells from patients with acute myeloid leukemia. Blood 97: 3882-3889, 2001.

26. Wulf GG, Wang R-Y, Kuehnle I, et al. A leukemic stem cell with intrinsic drug efflux capacity in acute myeloid leukemia. Blood 98: 1166-1173, 2001.

27. Palaiologou D, Panayiotidis P, Papanikolaou G, et al. Expression of three different ATP-binding cassette transporters and correlation to chemoresistance in acute myeloid leukemia. Int J Lab Hematol 37: 7-10, 2015.

\section{Correspondence:}

Mohammad Reza KERAMATI, MD

Cancer Molecular Pathology Research Center

Mashhad University of Medical Sciences,

MASHAD /IRAN

Tel: +985138521400

Fax: +985138591057

e-mail: keramatimr@mums.ac.ir

tayebe.sohrabi68@gmail.com 